\title{
Namur Modul Type Package - Definition
}

Beschreibungsmittel für die Automation modularer Anlagen

Die Modularisierung von Prozessanlagen wird als erfolgversprechende Möglichkeit angesehen, die künftigen Anforderungen an die Prozessindustrie zu bewältigen. Mit dem Projekt Dezentrale Intelligenz für modulare Anlagen (Dima) hat Wago auf der Namur-Hauptsitzung 2014 einen Weg gezeigt, wie sich die Anforderungen, die in der NE148 formuliert worden sind, erfüllen lassen. Bisherige Arbeiten ergaben, dass die Verwendung von Module Type Packages (MTP) zur Beschreibung von Prozessmodulen eine vielversprechende Lösung darstellt. Der Namur-Arbeitskreis AK 1.12 arbeitet derzeit, in Zusammenarbeit mit verschiedenen Ad-Hoc-Arbeitskreisen der Namur und dem ZVEI, an der Standardisierung des Namur-MTP. Dieser Beitrag gibt eine Übersicht über die Arbeiten der Arbeitsgruppen. Die Fragestellungen und Anforderungen der Arbeitskreise für Zustandsbasierte Prozessführung, Bedienbilder und Diagnose und Wartung modularer Anlagen werden skizziert. Ferner wird eine mögliche Architektur des Namur-MTP vorgestellt.

\section{SCHLAGWÖRTER Modularisierung / dezentrale Intelligenz / Prozessmodul / Prozessführungsebene}

\section{Namur Module Type Package - Definition - Describing the automation of modular plants}

Modularization of process plants has been identified as a crucial factor to fulfil future requirements in the process industry. With the 'Dima' project, presented at the Namur General Assembly 2014, Wago showed how the requirements of NE148 regarding modularization of process plants can be met. It has already been shown that Module Type Packages (MTP) offer a promising way to describe process modules. The Namur working group 1.12 is currently working on MTP standardization, together with several ad-hoc working groups of Namur and the ZVEI. This article presents the current situation regarding state-based control, human-machine interaction, and the diagnosis and maintenance of modular plants. In addition, a possible architecture of the Namur MTP is shown.

KEYWORDS modularisation / decentralised intelligence / process module / process control level 
JENS BERNSHAUSEN, Invite

AXEL HALLER, ABB

THOMAS HOLM, Wago Kontakttechnik

MARIO HOERNICKE, ABB Forschungszentrum

MICHAEL OBST, Technische Universität Dresden

JAN LADIGES, Helmut-Schmidt-Universität Hamburg

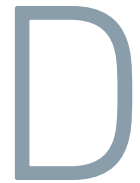

ie Anforderungen des Marktes an die Produktion in der Prozessindustrie haben sich in den vergangenen Dekaden verändert. Diese Änderungen sind geprägt durch schwankende Beschaffungs- und Absatzmärkte [1] sowie kürzere Produktlebenszyklen, insbesondere chemischer und pharmazeutischer Produkte [2]. Des Weiteren werden immer kürzere Innovationszyklen gefordert, und der Bedarf an kundenspezifischer Spezialisierung der Produkte steigt [3]. Umso wichtiger ist es, dass Produkt- und Technologieinnovationen schnell Marktreife erreichen und monetär zu verwerten sind. Ein früher Markteintritt neuer Produkte wird als entscheidender Faktor für den wirtschaftlichen Erfolg einer Produktion angesehen [4].

Unter anderem lässt sich daraus schlussfolgern, dass Produktionsanlagen die Produktionsmenge und das Produktportfolio entsprechend den aktuellen Marktbedingungen anpassen müssen. Einem Anstieg der Kapazitätsflexibilität steht im konventionellen Anlagenbau jedoch ein Verlust an Produktionseffizienz gegenüber [5]. Weder die kontinuierliche Produktion noch die Chargenproduktion sind derzeit durch den konventionellen Anlagenbau auf diese Marktbedingungen ausgerichtet. Folglich sind produzierende Unternehmen gezwungen, höhere Produktionsverluste hinzunehmen, um ein möglichst optimales Preis-Absatz-Verhältnis zu erreichen. Entsprechend werden neue Produkte lediglich für verlässliche Märkte eingeführt und neue, vielversprechende Technologien werden mit hoher Verzögerung angewandt [5].

Ortsbezogene Produktionskosten schwanken, während aufstrebende Länder ihren Produktionsindex erhöhen [1]. Weltweite politische Unsicherheiten sorgen dafür, dass ein Produktionsstandort, der während der Anlagenplanung noch als optimal identifiziert wurde, eventuell im Laufe der operativen Phase der Anlage nicht mehr den Anforderungen des Anlagenbetreibers entspricht. Der Bedarf an Mobilität von Produktionsanlagen zählt somit zu den Schlüsselfaktoren des zukünftigen Produktionserfolges [3].

Als eine Schlüsseltechnologie zur Überwindung dieser Herausforderungen, zumindest im Bereich kleinskaliger Prozessanlagen, wird die Modularität angese- hen [6]. Der Ansatz der Modularisierung stellt einen Schritt zur Beschleunigung von Bau und Planung von Produktionsanlagen kleiner bis mittlerer Größe dar [2]. Durch die Verwendung von branchenspezifischen Modulen, die fertige, in sich geschlossene funktionale Einheiten bilden, lässt sich der Engineeringaufwand für Prozessanlagen erheblich reduzieren [7]. Die Wiederverwendung von bewährten Modullösungen vermeidet Fehler in frühen Phasen des Engineerings, was wiederum zu Kosten- und Zeitersparnissen führt [8]. Neben der erstrebten Skalierbarkeit durch Hinzu- und Wegnahme von Modulen (numbering-up) wird auch eine bessere Datenbasis zur Kostenschätzung für die Planung und Errichtung der Anlagen geschaffen, da sich bekannter Aufwand lediglich in neuer Zusammensetzung wiederholt [9].

\section{ARBEITSKREISE ZUR AUTOMATISIERUNG MODULARER ANLAGEN}

Mit Dima (Dezentrale Intelligenz für modulare Anlagen) $[17,18]$ wurde von Wago eine herstellerunabhängige Methodik für die zentrale Herausforderung bei der Modularisierung von Produktionsanlagen der Prozessindustrie erarbeitet [7]: die flexible Konfiguration des Automatisierungssystems. Da das Engineering und der Betrieb modularer Anlagen von aktuellen Prozessleitsystemen (PLS) nicht hinreichend unterstützt werden $[10,12]$, musste von bekannten Strukturen des Automatisierungssystems abgewichen werden. Mit dieser Methodik werden wandlungsfähige modulare Produktionsanlagen unter Nutzung von zusätzlichen Steuerungen innerhalb der Module ermöglicht. Die heute zur Verfügung stehende Funktionalität der Automatisierungspyramide wird dennoch weiter nutzbar sein. Damit können die Investitionskosten bei Neu- und Umbau von Produktionsanlagen niedrig gehalten werden. Erarbeitet wurde die DimaMethodik von der Technischen Universität Dresden und der Helmut-Schmidt-Universität Hamburg in Zusammenarbeit mit der Firma Wago. 
Auf der Namur-Hauptsitzung 2014 stellte Wago die Dima-Methodik erstmals vor. Die damalige prototypische Umsetzung verwendete Simatic WinCC von Siemens als Bedien- und Beobachtungsfunktionalität sowie das Batch-Werkzeug Proficy Batch von General Electric zur Integration der vom Modul angebotenen Dienste und deren Orchestrierung. Bereits während der ersten Präsentation von Dima wurde angekündigt, die Ergebnisse dieser prototypischen Umsetzung den Gremien der Namur und des ZVEI zu übergeben, um darauf aufbauend ein herstellerneutrales Namur-MTP (Module Type Package) zur Beschreibung von Modulen zu erarbeiten.

\subsection{Struktur der Arbeitskreise}

Anfang 2015 wurden durch die Namur vier Ad-HocArbeitskreise aufgestellt, in denen sich Namur- und ZVEI-Mitglieder zusammengefunden haben, um gemeinsam eine MTP-Spezifikation zu erarbeiten. Die Inhalte des MTP wurden aufgeteilt und die entstandenen Aspekte zur Bearbeitung an diese Ad-Hoc-Arbeitskreise übergeben. Zur Synchronisation der Ergebnisse, sowie deren Konsistenzsicherung, wurde als übergeordnete Instanz ein gemeinsamer Arbeitskreis Modulare Automation, bestehend aus dem Namur-AK 1.12 und dem ZVEI-Spiegel-AK Modulare Automation, gegründet, siehe Bild 1. Seitdem wurde innerhalb dieser Arbeitskreise intensiv an der Weiterentwicklung des Namur-MTP gearbeitet. Insgesamt sind mehr als 25 Unternehmensvertreter mit dem Ziel beteiligt, eine Namur-Empfehlung über Inhalte und Struktur des Namur-MTP zu erarbeiten.

Die Arbeitskreise bearbeiten jeweils die Struktur und den Inhalt eines Aspektes des MTP. Die Aspekte sind dabei von der Tauchnitzschen PLS-Torte [19] abgeleitet.

\subsection{Inhalte der Arbeitskreise}

Im Einzelnen kümmern sich die Ad-Hoc Arbeitskreise um folgende Themen siehe Bild 2:

1 | Namur-AK 2.3.1: Zustandsbasierte Prozessführung

2 | Namur-AK 2.6.1: Diagnose \& Maintenance modularer Anlagen

3 | Namur-AK 2.9.1: HMI für modulare Anlagen

Die drei Arbeitskreise liefern Anforderungen bezüglich der Information, die mit dem Namur-MTP zwischen Modul-Engineering und dem Engineering der Prozessführungsebene (PFE-Engineering) ausgetauscht werden soll. $\mathrm{Zu}$ erarbeiten, wie diese Inhalte in eine geeignete Struktur übersetzt werden und durch Beschreibungsmittel sinnvoll im Namur-MTP modelliert werden können, ist Aufgabe des AK 1.12.1.

Dazu wurden mehrere Beschreibungsmittel betrachtet und bewertet. Um einen offenen Austausch auf Basis eines bereits bekannten und verbreiteten Standards zu ermöglichen, sollte grundsätzlich das verwendete Beschreibungsmittel XML-basiert sein. Des Weiteren wurde erkannt, dass sich die Innovationszy-

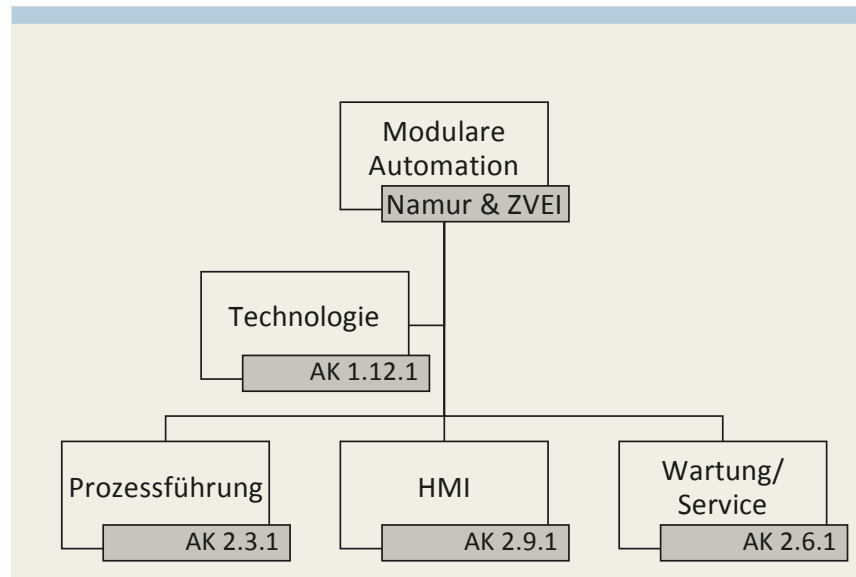

BILD 1: Organisatorische Struktur der Namur/ZVEI-Arbeitskreise

klen der einzelnen Aspekte stark unterscheiden. Daraus folgte der Beschluss, die Aspekte funktionsorientiert (zum Beispiel Bedienbild als eine Funktion, OPC-Schnittstellen als eine Funktion) zu betrachten und auch im Modell getrennt zu halten. Somit können entsprechende Bereiche innerhalb des Namur-MTP vereinfacht ausgetauscht werden, ohne das gesamte Namur-MTP zu verändern.

\section{KONZEPTE, ANNAHMEN, VORAUSSETZUNGEN MODULARER AUTOMATISIERUNG}

In Analogie zur physikalischen Modularisierung der Anlage [17] erfolgt eine funktionsorientierte Modularisierung. Dabei stellt ein Modul seine verfahrenstechnische Funktion als Dienst einer übergeordneten Prozessführungsebene (PFE) zur Verfügung. Es nimmt damit die Stellung eines Dienstanbieters ein. Die vom Modul angebotene Dienstleistung kann von der PFE abgerufen werden, die damit ein Dienstnutzer ist [23]. Die Integration mehrerer Module und deren Dienste zu einer Gesamtanlage wird als PFE-Engineering bezeichnet. Zur aufwandsarmen Umsetzung der so entstehenden dienstorientierten Architektur (service-oriented architecture, SOA) muss ein Modul mindestens folgende Grundfunktionalitäten der PFE unterstützen, das heißt auch die Modulbeschreibung muss eine Beschreibung folgender Schnittstellen beinhalten:

1 | Mensch-Maschine-Schnittstelle: Das Modul muss in der Lage sein, Daten zu erzeugen und zu übermitteln. Die Daten werden zur Anzeige und Bedienung der Anlage verwendet, wobei das Modul im PLS durch ein eigenes Bedienbild dargestellt wird.

2 | Steuern und Überwachen: Als Schnittstelle werden die internen Zustände des Moduls benötigt. Diese müssen durch das Modul ermittelt, über- 


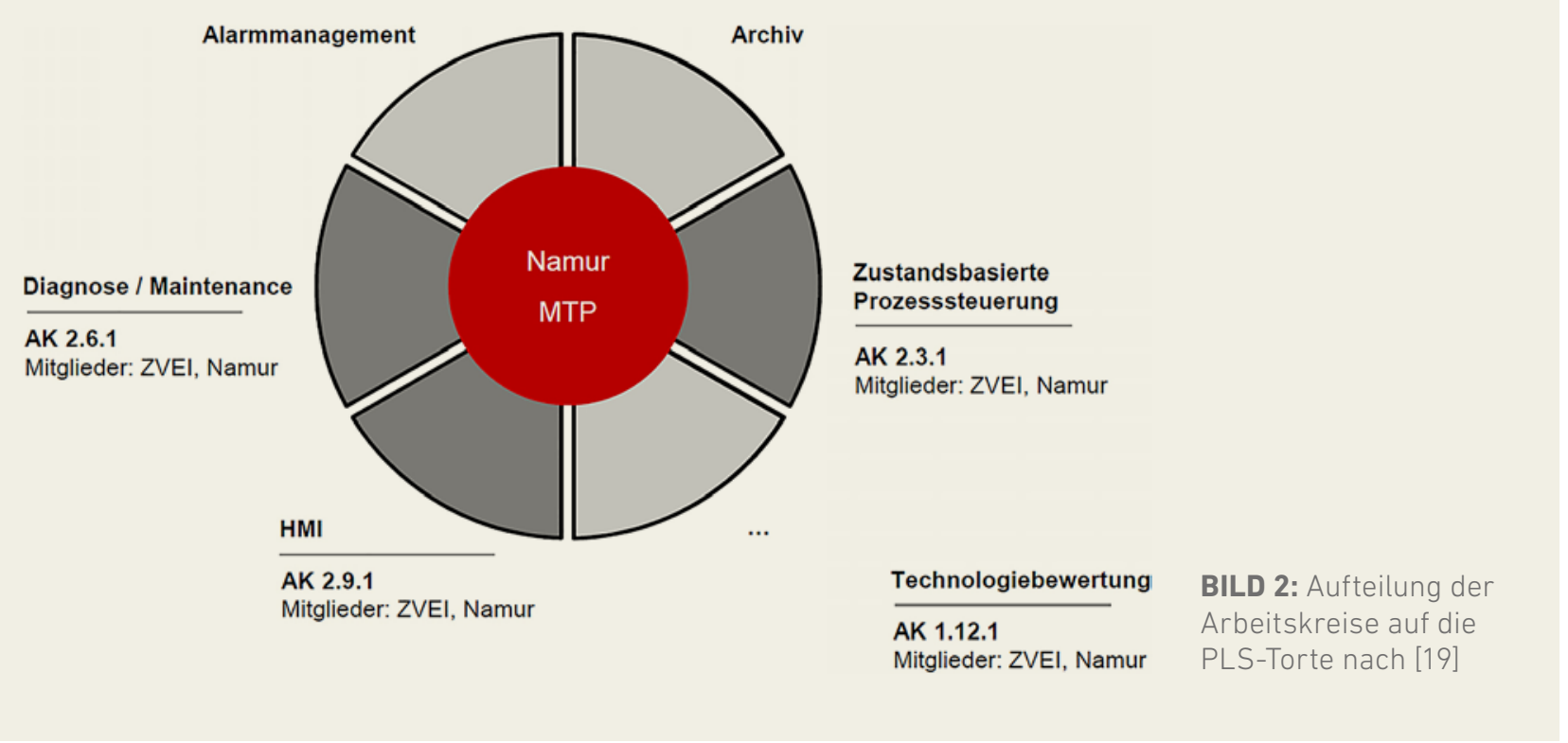

wacht und an die PFE übertragen werden. Zustandsinformationen des Moduls werden zur fehlerfreien und wunschgemäßen Abarbeitung von angeforderten Funktionen benötigt.

Des Weiteren wird im Folgenden angenommen, dass jedes Modul eine eigene Steuerung besitzt. Die nach NE 148 ebenfalls möglichen Module mit Remote I/O werden nicht weiter betrachtet. Um das dienstorientierte Konzept von Modulseite autark zu ermöglichen, ist dies eine Voraussetzung. Module mit Remote I/O können zwar auch dienstorientiert geführt werden, jedoch muss die notwendige Logik hierfür in der PFE abgebildet werden.

\subsection{Engineering-Workflow}

Bei der Betrachtung des Engineerings einer modularen Anlage muss grundsätzlich zwischen zwei Bereichen unterschieden werden:

1 | Modul-Engineering (Bild 3, linke Seite): Jeder Modultyp wird einmalig entwickelt. Dies beinhaltet die physikalische Gestaltung des hierin zu realisierenden Verfahrensschritts und die Erstellung der informationstechnischen Schnittstelle zu übergeordneten Systemen. Hinzu kommen das Engineering für die Steuerungslogik und die Bedienbilder. Letztlich wird das Engineering wie bei einer kleinen Anlage durchgeführt, mit dem Unterschied, dass das Modul in seiner Funktion generisch für verschiedene Einsatzarten ausgelegt werden muss. Zusätzlich zum physikalischen und informationstechnischen Entwurf des Moduls muss am Ende ein Namur-MTP zur Integration in die PFE generiert und mit dem Modul ausgeliefert werden. Hierfür gilt es, entsprechende Werkzeuge zu entwickeln.
2 | PFE-Engineering (Bild 3, rechte Seite): Beim PFE-Engineering werden die Module in die PFE integriert, indem die Namur-MTPs der Module in die PFE eingelesen und die nötigen Bestandteile auf PFE-Seite für jedes benötigte Modul generiert werden. Dies beinhaltet die Schnittstelle zum Informationsmodell des Moduls und die grafische Darstellung des Moduls. Zusätzlich wird das modulübergreifende Verfahren in der PFE konfiguriert. Die Prozedursteuerung zum zeitgerechten Abrufen und Überwachen (Orchestrierung) der Moduldienste wird erstellt. Die Ansteuerung der Dienste der Module wird parametriert und gegebenenfalls modulübergreifende Verriegelungslogik erzeugt. Letztlich wird die physikalische Kommunikation im Netzwerk-Engineering abgebildet und parametriert.

Dabei sind die Engineering-Prozesse des Modulherstellers (Modul-Engineering) und des Anwenders (PFE-Engineering) voneinander entkoppelt. Der Mehrwert der Modullieferanten liegt in der Mehrfachanwendung seiner Modultypen, womit ein kostenintensiveres Modul-Engineering gerechtfertigt ist. Der Mehrwert des Modulanwenders liegt in der verringerten Engineeringzeit für die Gesamtanlage. Hierdurch wird eine Win-win-Situation für beide Parteien geschaffen.

\subsection{PFE-Integration}

Werden mehrere Module zu einer Anlage kombiniert und physikalisch mit einem Backbone verbunden (Bild 3, rechte Seite), entsteht die Notwendigkeit, diese Module im Rahmen des PFE-Engineerings in eine übergeordnete PFE zu integrieren, siehe Bild 4. 


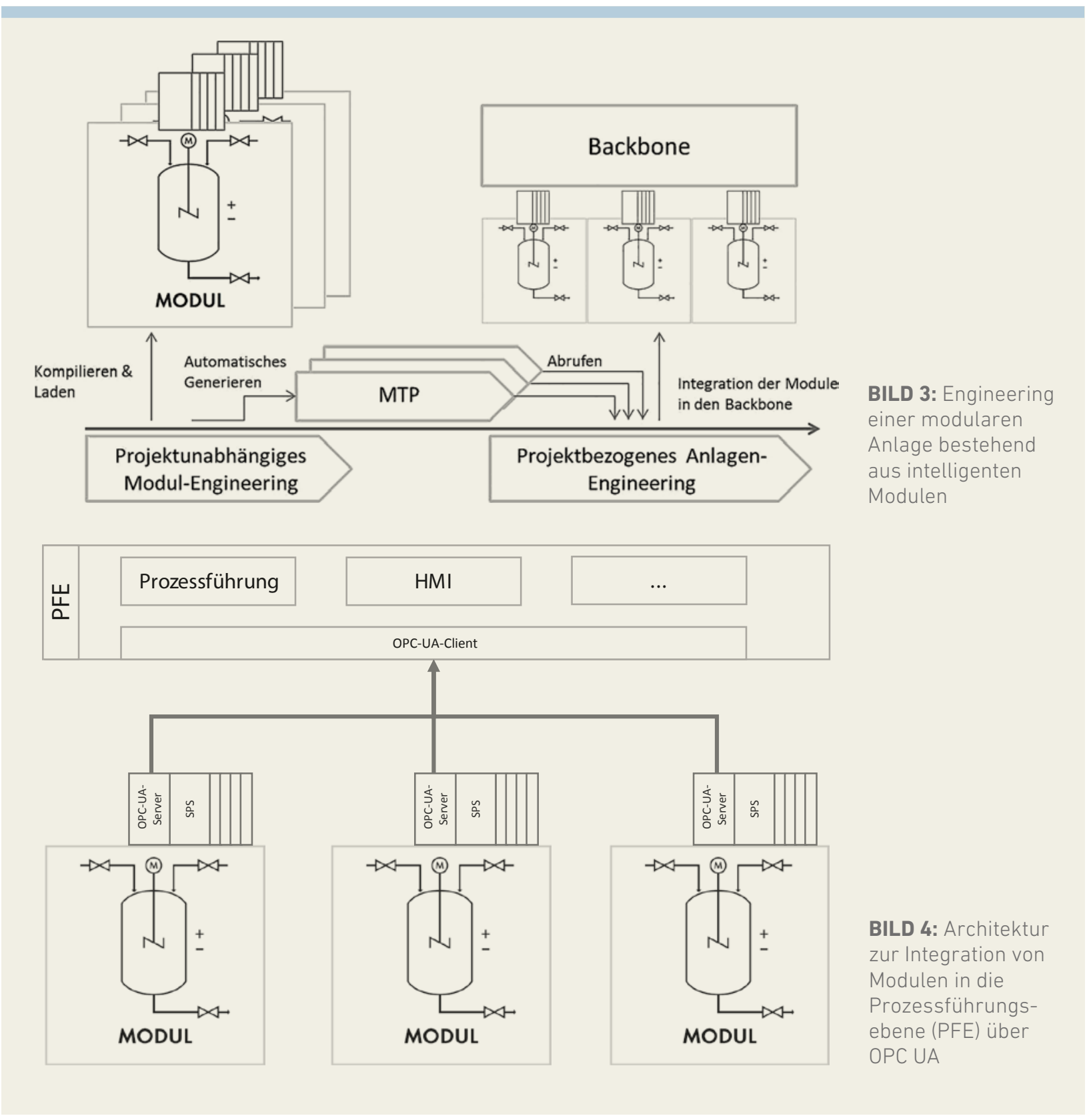

In der PFE kann beispielsweise ein Prozessleitsystem eingesetzt werden. Wie in [17] demonstriert, kann sich diese PFE aber ebenso aus Komponenten (wie Scada und Batch) zusammensetzen, die jeweils Teilfunktionalitäten eines Prozessleitsystems übernehmen.

Im Rahmen der im Beitrag beschriebenen Weiterentwicklung der Dima-Methodik wurde im Vergleich zu [18] das Kommunikationsmittel überarbeitet. Als Kommunikationstechnologie soll künftig OPC UA eingesetzt werden, wobei sich der OPC-UA-Server des Moduls direkt auf der Steuerung des Moduls beziehungsweise im Modul befinden sollte und durch den Modulhersteller erstellt und parametriert wird. In diesem ist dann alle für die Kommunikation notwendige Information abgebildet.

\subsection{Zustandsbasierte Prozessführung}

Um die Dienste der einzelnen Module in eine für die Produktion des gewünschten Produkts erforderliche Reihenfolge zu bringen (Orchestrierung), muss zum Beispiel bei einem kontinuierlich betriebenen Reaktionsprozess das Anfahren des Reaktors mit dem Vorlegen der Ausgangsprodukte abgestimmt werden. Da diese zusätzliche Orchestrierungsfunktion erst durch Kombination verschiedener Module notwendig wird, muss diese von einer noch während des PFEEngineering gestaltbaren Automatisierungsinstanz, zum Beispiel dem übergeordneten Leitsystem, übernommen werden.

Zur Sicherstellung der modulübergreifenden Dienstorchestrierung ist im Leitsystem die Kenntnis der ak- 
tuellen Zustände, beispielsweise Run, Stop oder Fehler, und entsprechender Zustandsübergänge der Module beziehungsweise der Moduldienste notwendig. Diese Information wird durch die dezentrale Intelligenz eines jeden Moduls ermittelt und über die Kommunikationsschnittstelle zur Verfügung gestellt. Die Definition der Zustände muss hersteller- und modulunabhängig und somit einheitlich über alle Module und Dienste hinweg geschehen.

Die IEC 61512 [15] beinhaltet eine exemplarische Definition von Zuständen und legt die Bedingungen der möglichen Zustandsübergänge für Prozedurelemente fest, siehe Bild 5. Im aktuellen Namur/ZVEIProjekt stellt diese Norm eine gute Arbeitsgrundlage für die Zustandsbeschreibung und -steuerung von Modulen und Diensten dar. Es bleibt allerdings noch zu prüfen, ob weitere Zustände, Zustandsübergänge und/oder Nebenbedingungen für Zustandsübergänge für die Steuerung von Modulen und Diensten notwendig sind. Eine zustandsbasierte Steuerung findet in der chemischen Industrie bereits heute Anwendung. So wird in [26] beispielsweise beschrieben, wie ein konventioneller Prozess in Module zerlegt wird und anschließend für die Module Automaten zur Steuerung beschrieben und implementiert werden.

Wie schon beschrieben, werden die in den Modulen vorgesehenen prozesstechnischen Funktionen als Dienste gekapselt. Ein Reaktormodul mit einem Mixer beispielsweise könnte demnach den Dienst Mischen anbieten. Da die Edukte in den Reaktor einzufüllen sind, wird vom Reaktor des Weiteren der Dienst Befüllen angeboten, der sich je nach Anzahl und Benennung der Einfüllstutzen in zum Beispiel Befüllen $A$ und Befüllen $B$ unterscheiden kann. Verfügt der Reaktor über ein Heizsystem, kann ebenfalls der Dienst Heizen ausgeführt werden. Ein entsprechender Parametersatz dieses Dienstes könnte die Zieltemperatur, die Temperaturanstiegsgeschwindigkeit und die Haltezeit sein. Eine detaillierte Betrachtung der Dienstschnittstelle und deren Abbildung auf den Namur-MTP sowie des Integrations-Workflows kann den Beiträgen [20] und [21] entnommen werden.

\subsection{Bedienerschnittstelle für modulare Anlagen}

Die zentrale Herausforderung der Bedien- und Beobachtbarkeit des über mehrere Module verteilten Prozesses ist die automatische Bedienbilderstellung und die Realisierung des nach [11] formulierten einheitlichen Look and Feel einer modularen Anlage.

Da der Modulhersteller für die Planung, den Aufbau und die Programmierung des Moduls verantwortlich ist, fertigt er ebenso das oder die Bedienbild(er) des Moduls an. Eine Kenntnis der in industriellen Anlagenprojekten meist projektspezifisch verwendeten Bedienbildbibliothek der übergeordneten PFE hat er zu diesem Zeitpunkt jedoch nicht. Die Generierung des modulspezifischen Bedienbilds in der PFE der Gesamtanlage kann somit erst nach Integration des Moduls, im PFE-Enginee-

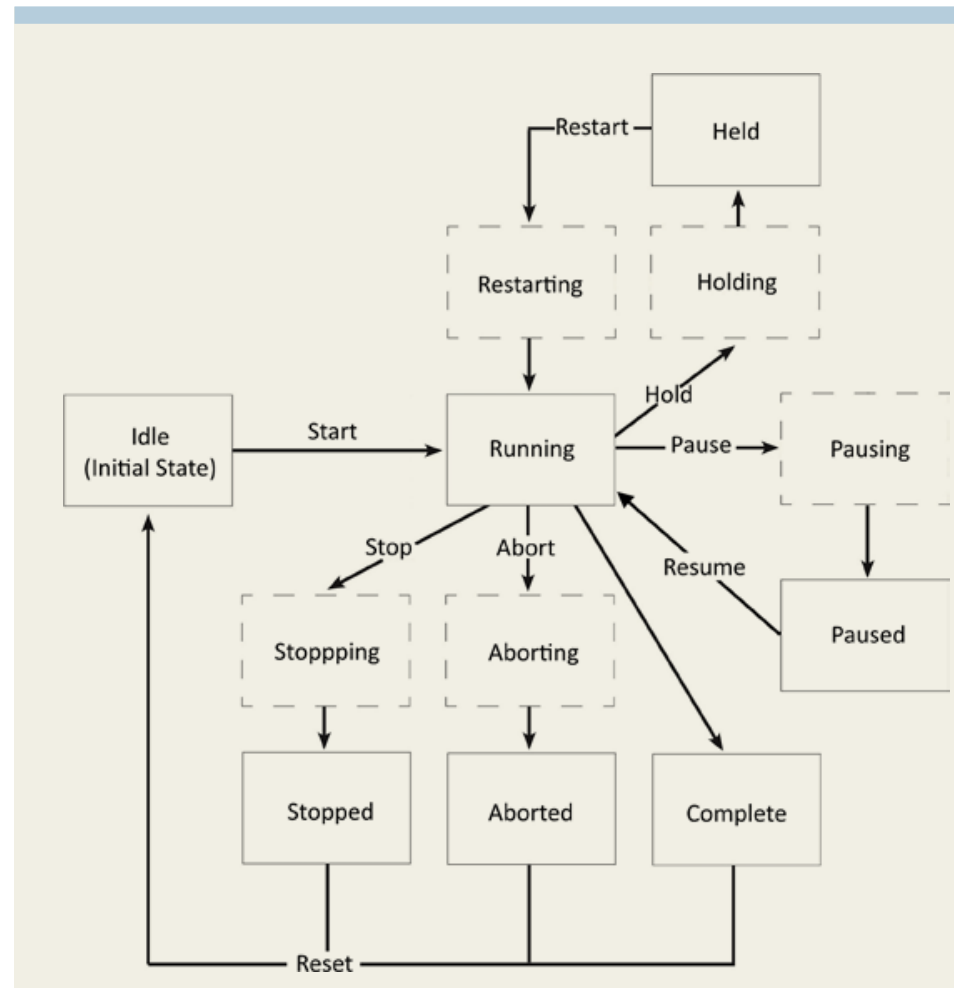

BILD 5: Beispielautomat der IEC 61512 [13]

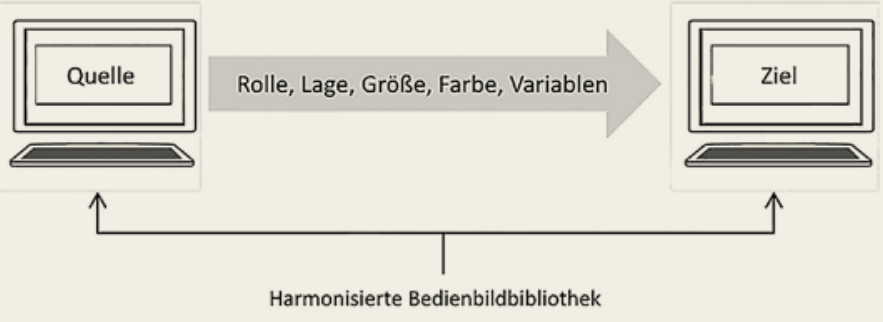

BILD 6: Bedienbildbeschreibung unter Nutzung einer Bedienbildbibliothek

ring, erfolgen. Erst zu diesem Zeitpunkt ist die Bedienbildbibliothek einheitlich festgelegt.

Zur Umsetzung der modulspezifischen Bedienbilder in projektspezifisch vereinheitlichte Bedienbildelemente müssen die Bedienbilder in einer darstellungsunabhängigen Beschreibungsform vorliegen. Diese Beschreibung enthält die Information zum Typ des darzustellenden Prozessequipments, dessen Lageund Größeninformation und gegebenenfalls Farbinformation für dynamische Anzeigeeffekte, siehe Bild 6. Diese Information ist durch einen Algorithmus zugänglich, der die projektabhängigen Bedienbildelemente in gewünschter Darstellung und Lage auf das Bedienbild in der PFE setzt. Die dynamischen Daten im Bedienbild werden anschließend mit den im MTP definierten Kommunikationsvariablen zur Modulsteuerung verknüpft [15]. 


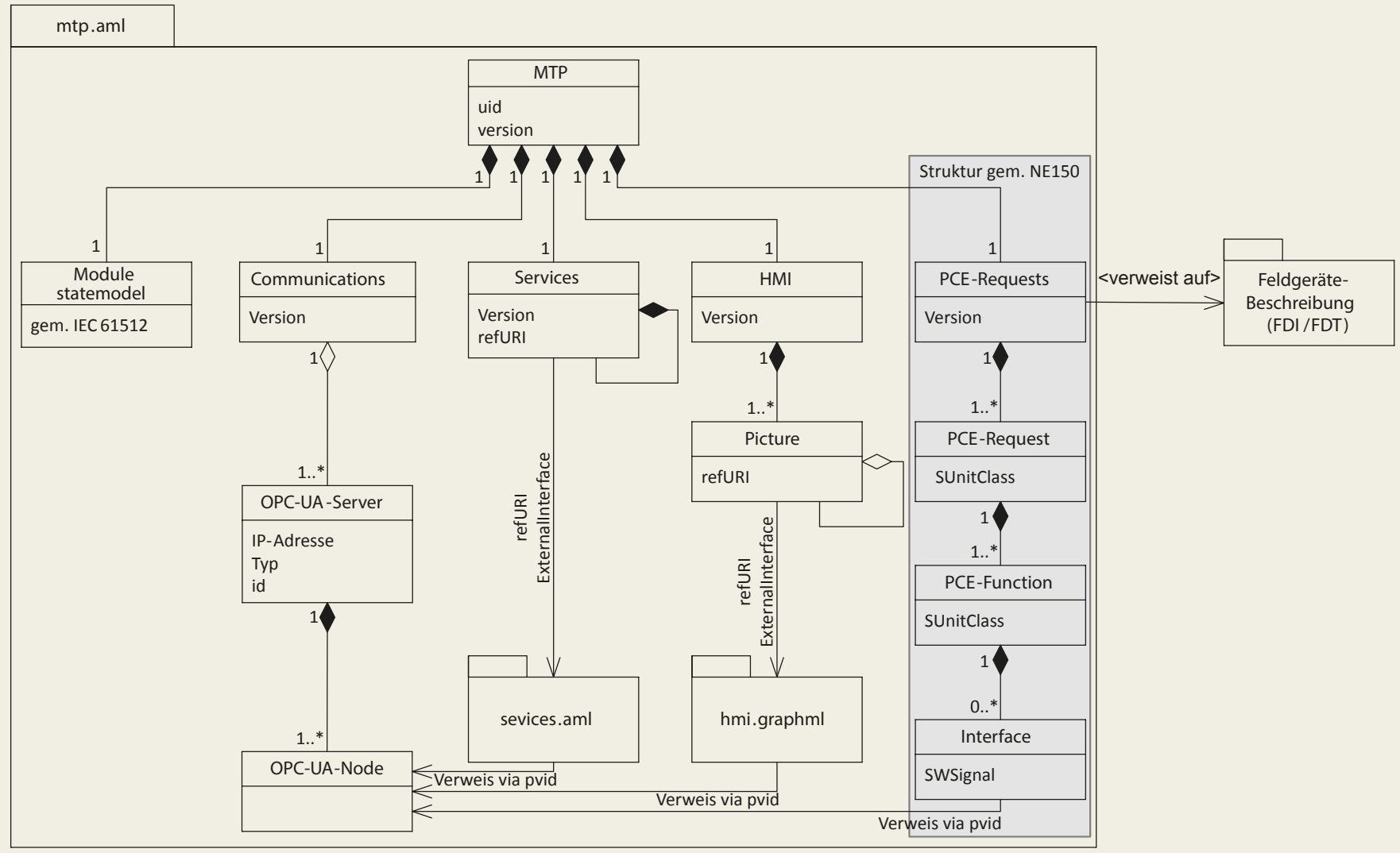

BILD 7: Architektur des Manifests mit den Aspekten Zustandsmodell, Kommunikation, Diensten und HMI

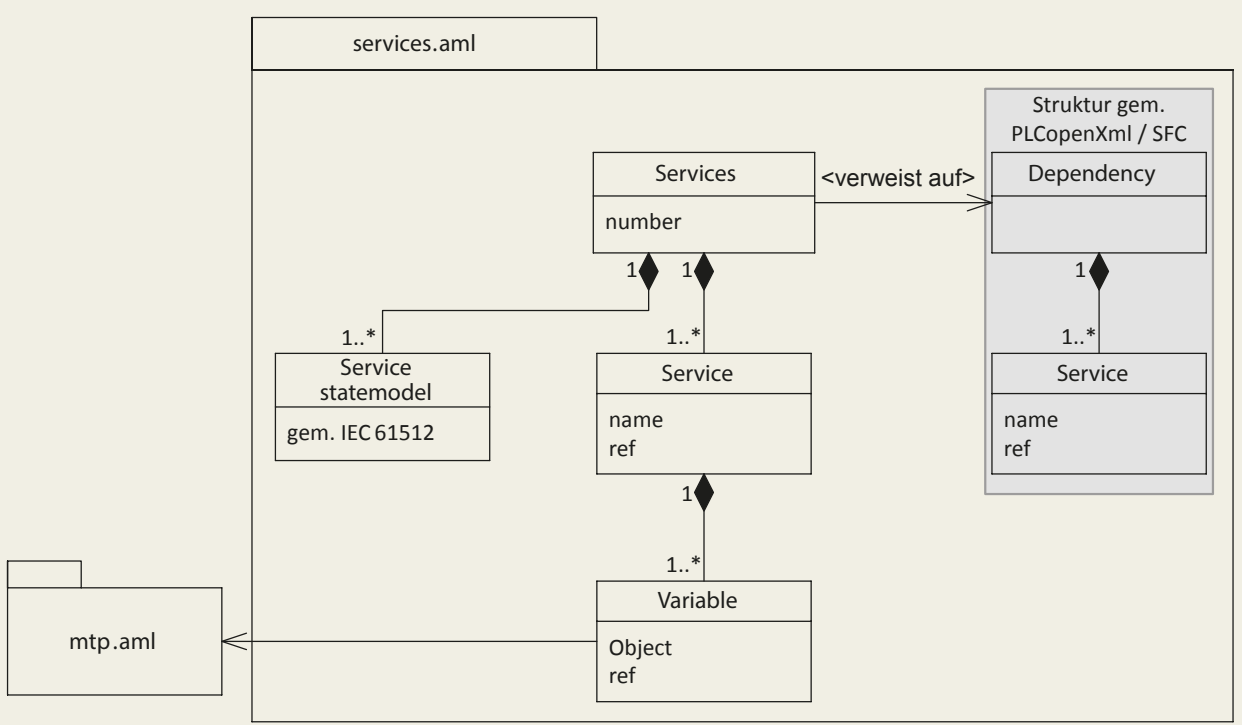

BILD 8: Architektur der Dienstbeschreibung 


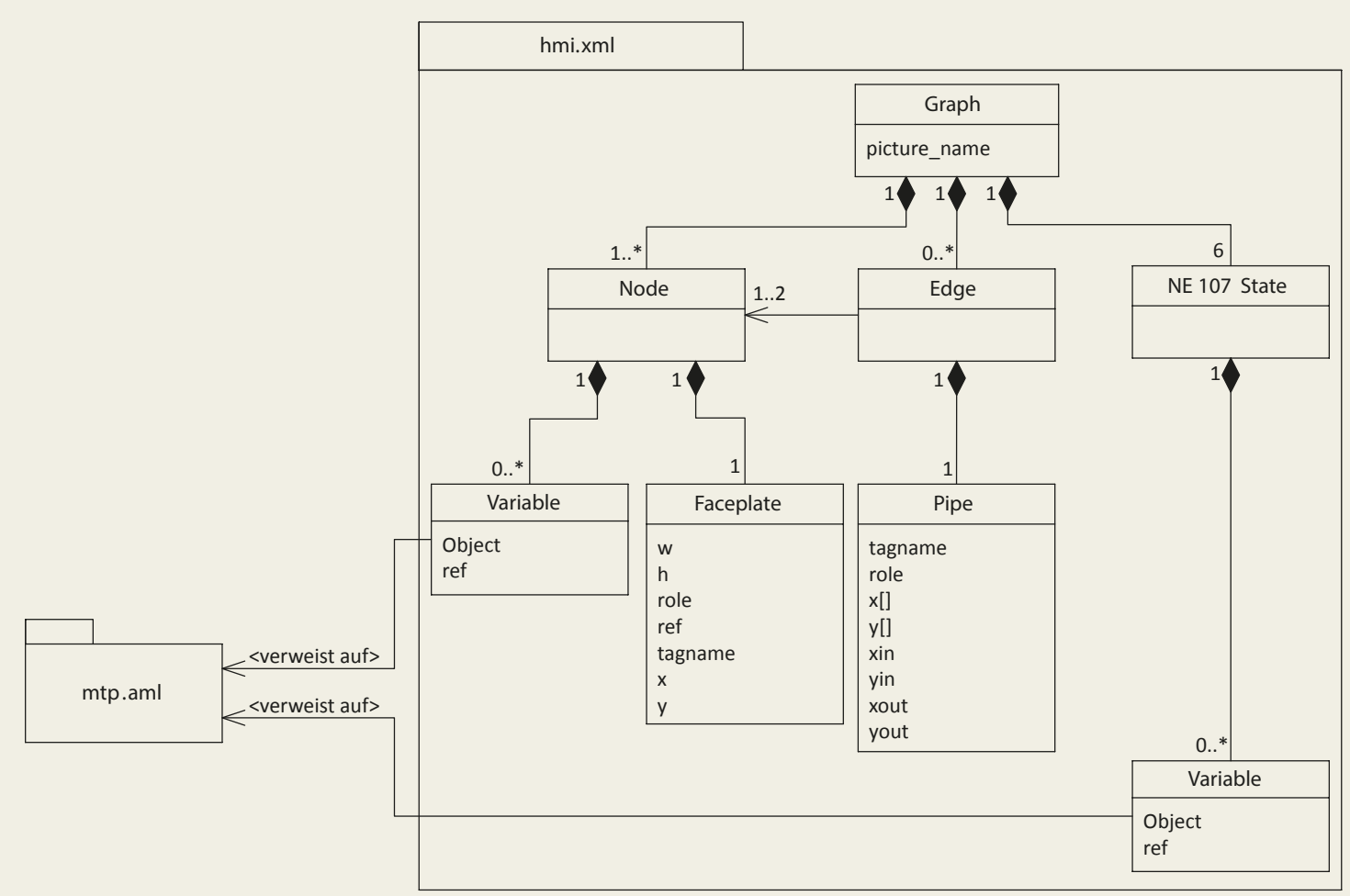

BILD 9: Architektur der mit GraphML modellierten Bedienbildbeschreibung

Die Nutzung eines Bibliothekskonzepts bedingt, dass die Bibliothek im Engineeringwerkzeug des Modulherstellers und im Engineeringwerkzeug für die PFE vorliegt. Die zur Verwendung der Bibliothek zu übertragende Information ist in Bild 6 dargestellt. Ein Vorschlag für eine entsprechende Bibliothek wird zurzeit im Ad-Hoc-AK 2.9.1 erstellt.

Alle Information, die im Modul-Engineering erarbeitet und während des PFE-Engineerings benötigt wird, wird in einem Informationsträger, dem Namur-MTP abgelegt. Mit Hilfe dieses Informationsträgers kann die PFE entsprechend konfiguriert werden.

\section{ARCHITEKTUR DES NAMUR-MTP}

Die bisherigen Arbeiten der Arbeitskreise folgten einem funktionsorientierten Ansatz. Die Modelle innerhalb des Namur-MTP sind deshalb weitgehend entkoppelt voneinander zu betrachten, was den Vorteil hat, dass diese verhältnismäßig einfach durch neuere Versionen ausgetauscht werden können. Das Namur-MTP ist letztlich eine Zipdatei, die eine Ordnerstruktur beinhaltet, die alle notwendigen Aspekte des Moduls in Form von weiteren Dateien definierter Typen enthält. Übergeordnet, als Einstiegspunkt für eine Interpretation zu betrachten, wird eine mtp.aml erzeugt, die die einzelnen Teile des Namur-MTP miteinander verbindet.

\subsection{Inhalte der Organisationsdatei}

Das MTP-Manifest (mtp.aml) wurde in AutomationML modelliert. Dieses Beschreibungsmittel ist in IEC 62714 [16] standardisiert und durch seinen MultiBibliotheksansatz geeignet, eine Vielzahl von Aspekten abzubilden.

Das Manifest enthält, neben der Version, Herstellerinformation und einer eindeutigen MTP-ID, Links zu den Beschreibungsdateien folgender Aspekte, siehe Bild 7:

- Das Zustandsmodell des Moduls beziehungsweise aller Dienste: Jedes Modul implementiert wenigstens eine Funktion. Die PFE muss über den aktuellen Zustand der Funktion(en) Bescheid wissen und ungewünschte Überschneidungen im Prozessablauf verhindern (zum Beispiel gleichzeitiges Heizen und Kühlen desselben Behälters).

- Beschreibung der Kommunikationsschnittstelle des Moduls: Das Modul wird von der PFE über die moduleigene Kommunikationsschnittstelle angesprochen. Im Falle eines integrierten OPC-UA-Servers ist beispielsweise die URL und die IP-Adresse notwendig. Diese Information muss im Manifest vorhanden sein, wobei integrationsabhängige Parameter (zum Beispiel IP-Adresse) selbstverständlich nachträglich während des PFE-Engineerings ergänzt werden dürfen. 
- Alle Dienste, die das Modul der PFE zur Verfügung stellt: Üblicherweise implementieren Module mehrere Funktionen, wie Rühren und Temperieren, als separate Dienste. Diese können durch die PFE in die übergeordnete Prozessteuerung integriert werden.

- Beschreibung der Bedienerschnittstelle: Die Bedienbildhierarchie innerhalb des Moduls und Verweise auf die Bedienbildbeschreibungen müssen dem PFE bekannt gegeben werden. Deshalb werden aus dem Manifest Verknüpfungen zu den zugehörigen Beschreibungen bereitgestellt.

- PLT-Stelleninformation (PCE-Request): Wenn die internen PLT-Stellen des Moduls der PFE bekannt gegeben werden sollen, so muss ein Link auf die zugehörige Beschreibungsdatei im Manifest vorhanden sein.

Die Merkmale der Aspekte Services und HMI verweisen jeweils auf eine beziehungsweise mehrere Dateien in der Ordnerstruktur des MTP. Beispielsweise werden die Dienste eines Moduls in der Datei services.aml (zeigt dessen interne Struktur) abgebildet. Jeder Dienst verfügt über ein eigenständiges Zustandsmodell. Für die Beschreibung von Abhängigkeiten zwischen verschiedenen Diensten (zum Beispiel gleichzeitiger Ausschluss, Abfolgen) ist ein Bereich Dependency vorgesehen, in dem eine Verhaltensbeschreibung mit PLCopenXML [21] formal abgebildet wird.

\section{REFERENZEN}

[1] CEFIC The European Chemical Industry Council: The european chemical industry - Facts and Figures. http://www.cefic.org/Facts-and-Figures/, 2014

[2] Bramsiepe, C.; Schembecker, G.: Die 50 \%-Idee: Modularisierung im Planungsprozess. Chemie Ingenieur Technik, vo84(5), S. 581-587, 2012

[3] ZVEl e.V: Life-Cycle-Management für Produkte und Systeme der Automation: Ein Leitfaden des Arbeitskreises Systemaspekte im ZVEI Fachverband Automation. 2010.

[4] PAAT Team Tutzing, ProcessNet: Die $50 \%$ Idee: Vom Produkt zur Produktionsanlage in der halben Zeit. Thesen Tutzing, ProcessNet, 2009

[5] Bramsiepe, C.; Sievers, S.; Seifert, T.; Stefanidis, G.D.; Vlachos, D.G.; Schnitzer, H.; Muster, B.; Brunner, C.; Sanders, J.P.M.; Bruins, M.E.; Schembecker, G.: Low-cost small scale processing technologies for production applications in various environments - Mass produced factories. Chemical Engineering and Processing: Process Intensification 51, S. 32-52, 2012

[6] Obst, M.; Holm, T.; Bleuel, S.; Claussnitzer, U.; Evertz, L.; Jäger, T.; Nekolla, T:: Automatisierung im Life Cycle modularer Anlagen: Welche Veränderungen und Chancen sich ergeben. atp edition - Automatisierungstechnische Praxis 55(1-2), S. 24-31, 2013

[7] Urbas, L.; Bleuel, S.; Jäger, T.; Schmitz, S.; Evertz, L.; Nekolla, T: Automatisierung von Prozessmodu-len: Von Package-Unit Integration zu modularen Anlagen. atp edition - Automatisierungstechnische Praxis 54(1-2), S. 44-53, 2012

[8] Hady, L.; Wozny, G.: Multikriterielle Aspekte der Modularisierung bei der Planung verfahrenstechnischer Anlagen. Chemie Ingenieur Technik 84(5), S. 597-614, 2012

[9] Hady, L.; Dylag, M.; Wozny, G.: Kostenschätzung und Kostenkalkulation im chemischen Anlagenbau. Czasopismo Techniczne z.(5-M), S. 159-176, 2008

[10] Fay, A.; Drumm, 0.; Eckardt, R.; Gutermuth, G.; Krumsiek, D.; Löwen, U.; Schertl, A.; Schindler, T.; Schröck, S.: Anforderungen an Leitsysteme durch Industrie 4.0. In: Tagungsband Automation 2014, [CD]. VDI, 2014
[11] NE 148: Anforderungen an die Automatisierungstechnik durch die Modularisierung verfahrenstechnischer Anlagen. Namur 2013

[12] Urbas, L.: Zentrales Leitsystem ja oder nein?. process-online: http://www.process.vogel.de/automatisierung_prozessleittechnik/ articles/451796/?cmp=nl-98; zuletzt: 21.07 .2014

[13] DIN EN 61512-1: Chargenorientierte Fahrweise. 2010

[14] Hawkins, W.; Brandl, D.: Applying ISA-88 in discrete and continuous manufacturing. Momentum Press, 2010

[15] Urbas, L.; Doherr, F.: autoHMI: a model driven software engineering approach for HMls in process industries. IEEE Int. Conf. Computer Science and Automation Engineering, 2011

[16] IEC 62714: Datenaustauschformat für Planungsdaten industrieller Automatisierungssysteme-AutomationML, 2013

[17] Holm, T.; Obst, M.; Fay, A.; Urbas, L.; Albers, T.; Kreft, S.; Hempen, U.: Dezentrale Intelligenz für modulare Automation. atp edition Automatisierungstechnische Praxis 56(11), S.34-43, 2014.

[18] Obst, M.; Holm, T.; Urbas, L.; Fay, A.; Kreft, S.; Hempen, U.; Albers, T.: Beschreibung von Prozessmodulen. atp edition Automatisierungstechnische Praxis 57(1-2), S. 48-59, 2015

[19] Tauchnitz, T.: Die „neuen Prozeßleitsysteme - wohin geht die Reise?" atp edition - Automatisierungstechnische Praxis 38(11), S. $12-23,1996$

[20] Diedrich, C.; Meyer, M.; Evertz, L.; Schäfer, W.: Dienste in der Automatisierungstechnik. atp edition - Automatisierungstechnische Praxis 56(12), S. 24-35, 2014

[21] PLCopen: PLCopen TC6 XML. www.plcopen.org [Abruf: 2015-11-04]

[22] GraphML Team: The GraphML File Format. http://graphml.graphdrawing.org [Abruf: 20.01.2015]

[23] David A. Huffman: Benefits of State based Control. White Paper veröffentlich in "Control Global". https://www.controlglobal.com/ assets/knowledge_centers/abb/assets/Benefits-of-statebased-control-white-paper.pdf

[24] Holm, T.; Obst, M.; Ladiges, J.; Urbas, L.; Fay, A.; Albers, T.; Hempen, U.: Namur Module Type Package - Implementierung, Anwendung des Namur-MTP für Prozessanlagen. atp edition - Automatisierungstechnische Praxis 58 (1-2), S. 82-90, 2016

[25] Hoernicke, M; Holm, T.; et al: Beschreibungsmittel für verfahrenstechnische Module - Eine Übersicht der Ergebnisse des Namur AK 1.12.1. Angenommen für den VDI-Kongress Automation 2016, Baden-Baden 
Der HMI-Aspekt ist aufgeteilt in Bedienbildhierarchie und Bedienbildbeschreibung. Die Bedienbildhierarchie ist Teil des MTP-Manifests (mtp.aml - vergleiche Bild 7). Die einzelnen Bedienbildbeschreibungen selbst sind in leicht austauschbaren Dateien in der Ordnerstruktur des MTP abgelegt. Als Modellierungssprache für die Bedienbilder, die sich aus Nodes (Knoten) - für das Prozessequipment: wie Ventile und Pumpen - und Edges (Kanten) - zur Abbildung von Rohrleitungen und Informationsflüssen - zusammensetzen, wurde das durch Werkzeuge und Bibliotheken gut unterstütze Format GraphML [22] gewählt. Die Architektur dieser Datei zeigt Bild 9.

Nodes entsprechen den Bedienbildelementen und besitzen neben ihrer Bedeutung auch Lage-, Größen- und Variableninformation. Nodes können durch Edges verbunden sein. Eine Edge kann somit als Rohrleitung oder Informationsfluss auf dem Bedienbild interpretiert werden. Um Diagnoseinformation des Moduls dem Bediener zugänglich zu machen, enthält jedes Bedienbild entsprechende Statusanzeigen mit Verbindungen zu den passenden Variablen. Letztlich wird das Bedienbild als Graph abgebildet und ist somit während des PFE-Engineerings verhältnismäßig einfach interpretierbar.

Sowohl die services.aml als auch die hmi.xml referenzieren auf Variablen, die im Manifest global beschrieben sind und damit für das Namur-MTP, beziehungsweise für den darin beschriebenen Modultyp, überall zugänglich sind.

\section{ZUSAMMENFASSUNG UND AUSBLICK}

Die gemeinsamen Arbeitskreise der Namur und des ZVEI beschäftigen sich seit Anfang 2015 mit der Definition der Inhalte und möglicher Technologien, um die Funktionalität von Package Units, Prozessmodellen und Teilanlagen herstellerneutral in übergeordnete Prozessführungsebenen zu integrieren. In den Bereichen HMI, zustandsbasierte Prozessführung sowie Diagnose\&Maintenance konnten bereits wichtige Ergebnisse erzielt werden. Es gilt zukünftig, diese weiter zu detaillieren und geeignete Engineeringschnittstellen zu schaffen, die eine Erzeugung und eine Integration des Namur-MTP ermöglichen.

In dem Beitrag [24], Namur Module Type Package Implementierung, Anwendung des Namur-MTP für Prozessanlagen in dieser Ausgabe der atp edition wird eine prototypisch implementierte Schnittstelle beschrieben, die den aktuellen Stand des Namur-MTP bereits integrieren kann. Zudem wird hier eine Validierung der geschilderten MTP-Inhalte und -Strukturen vorgestellt. Die diskutierten Formate und das über diesen Beitrag hinausgehende Gesamtkonzept des Namur-MTP sollen beim Branchentreffen der Automatisierungstechnik VDI-Kongress Automation 2016 im Namen von ZVEI und Namur präsentiert werden. Neben dem Namur-MTP und dessen Architektur soll die Schnittstelle zur Integration in eine PFE zur Laufzeit diskutiert werden [25].

\section{AUTOREN}

Dr.-Ing. JENS BERNSHAUSEN (geb. 1980) ist Mitarbeiter des Forschungszentrums Invite $\mathrm{GmbH}$ und ist dort zuständig für die Automatisierung modularer Produktionsanlagen. Seit Oktober 2014 leitet er den Namur-Arbeitskreis 1.12 Automatisierung modularer Anlagen.

Invite GmbH, Chempark Leverkusen, Geb. W32, D-51368 Leverkusen,

Tel. +49 (0) 21431203127 ,

E-Mail: bernshausen@invite-research.com

Dipl.-Ing. AXEL HALLER (geb. 1961) ist Technology und Portfolio Manager im Geschäftsbereich Öl, Gas \& Chemie Deutschland bei ABB Automation und seit Januar 2015 Vorsitzender des ZVEI Arbeitskreises Modulare Automation.

Dipl.-Ing. THOMAS HOLM (geb. 1979) ist Key-Account Manager der Wago Kontakttechnik, Minden. Sein Forschungsschwerpunkt liegt im effizienten Engineering von Automatisierungssystemen wandlungsfähiger Produktionsanlagen.

Dipl.-Ing. (FH) MARIO HOERNICKE (geb. 1984) ist Principal Scientist am ABB Forschungszentrum in Ladenburg. Sein Forschungsschwerpunkt umfasst die Entwicklung neuer und innovativer Konzepte im Bereich Virtuelle Anlage, Operations Management und modularer Automatisierungstechnik.

Dipl. Ing. MICHAEL OBST (geb. 1985) ist wissenschaftlicher Mitarbeiter der Professur für Prozessleittechnik an der Technischen Universität Dresden mit den Schwerpunkten: Informationsmodellierung und Unterstützungssysteme für modulares Engineering.

M.Sc. JAN LADIGES (geb. 1984) arbeitet seit Oktober 2012 als wissenschaftlicher Mitarbeiter am Institut für Automatisierungstechnik der Helmut-SchmidtUniversität Hamburg bei Professor Fay. Sein Forschungsschwerpunkt liegt in der automatisierten Bestimmung von Anlagenund Prozesseigenschaften evolvierender Fertigungsanlagen. 